Received: 26 September 2018

Accepted: 7 March 2019

Published online: 21 March 2019

\section{OPEN The pentaglycine bridges of Staphylococcus aureus peptidoglycan are essential for cell integrity}

João M. Monteiro ${ }^{1}$, Gonçalo Covas ${ }^{1,3}$, Daniela Rausch ${ }^{2}$, Sérgio R. Filipe $\mathbb{I}^{3,1}$, Tanja Schneider ${ }^{2}$, Hans-Georg Sahl ${ }^{2}$ \& Mariana G. Pinho ${ }^{1}{ }^{1}$

Bacterial cells are surrounded by cell wall, whose main component is peptidoglycan (PG), a macromolecule that withstands the internal turgor of the cell. PG composition can vary considerably between species. The Gram-positive pathogen Staphylococcus aureus possesses highly crosslinked PG due to the presence of cross bridges containing five glycines, which are synthesised by the FemXAB protein family. FemX adds the first glycine of the cross bridge, while FemA and FemB add the second and the third, and the fourth and the fifth glycines, respectively. Of these, FemX was reported to be essential. To investigate the essentiality of FemAB, we constructed a conditional $S$. aureus mutant of the $f e m A B$ operon. Depletion of $f e m A B$ was lethal, with cells appearing as pseudomulticellular forms that eventually lyse due to extensive membrane rupture. This deleterious effect was mitigated by drastically increasing the osmolarity of the medium, indicating that pentaglycine crosslinks are required for $S$. aureus cells to withstand internal turgor. Despite the absence of canonical membrane targeting domains, FemA has been shown to localise at the membrane. To study its mechanism of localisation, we constructed mutants in key residues present in the putative transferase pocket and the $\alpha 6$ helix of FemA, possibly involved in tRNA binding. Mutations in the $\alpha 6$ helix led to a sharp decrease in protein activity in vivo and in vitro but did not impair correct membrane localisation, indicating that FemA activity is not required for localisation. Our data indicates that, contrarily to what was previously thought, $S$. aureus cells do not survive in the absence of a pentaglycine cross bridge.

S. aureus is one of the main pathogens responsible for life-threatening infections worldwide, particularly hospitaland community-acquired methicillin resistant $S$. aureus strains (HA-MRSA and CA-MRSA, respectively), which constitute a major challenge to antibiotic therapy ${ }^{1,2}$. Most of the widely used, and more potent antibiotics, target steps in the biosynthesis of peptidoglycan (PG), the core component of the bacterial wall. PG is a macromolecule composed of glycan chains, where each unit is constituted of $N$-acetylmuramic acid (MurNAc) and $\mathrm{N}$-acetylglucosamine (GlcNAc) sugars, with a stem peptide attached to MurNAc. Glycan chains are connected (crosslinked) through flexible species-specific peptide bridges, creating a mesh-like structure that envelops the $\mathrm{cell}^{3}$. The structural features of PG confer both robustness and flexibility to the cell envelope, which are necessary to withstand high pressure derived from intracellular turgor ${ }^{4}$.

MRSA strains are resistant to $\beta$-lactams, which irreversibly acylate the transpeptidase domain of Penicillin-Binding Proteins (PBPs), enzymes responsible for the last steps of PG biosynthesis ${ }^{1}$. In these strains, the major determinant of methicillin resistance is the acquired mecA gene, which encodes for PBP2A, an enzyme insensitive to $\beta$-lactam acylation ${ }^{5}$. However, high-level $\beta$-lactam resistance is in fact dependent on several additional elements, which were initially identified by transposon mutagenesis and termed fem (factor essential for methicillin resistance) or aux (auxiliary) genes ${ }^{6,7}$. Approximately $30 \mathrm{fem} / \mathrm{aux}$ determinants have been identified so far and most are housekeeping genes, involved in a variety of cellular processes and probably present in every S. aureus strain ${ }^{8}$. Three closely related factors - $f m h B$ and the co-transcribed femA and femB genes, encode for the

${ }^{1}$ Instituto de Tecnologia Química e Biológica António Xavier, Universidade Nova de Lisboa, Oeiras, Portugal. ${ }^{2}$ Institute of Pharmaceutical Microbiology, University of Bonn, 53115, Bonn, Germany. ${ }^{3}$ UCIBIO-REQUIMTE, Departamento de Ciências da Vida, Faculdade de Ciências e Tecnologia, Universidade Nova de Lisboa, Caparica, Portugal. Correspondence and requests for materials should be addressed to M.G.P. (email: mgpinho@itqb.unl.pt) 
FemX, FemA and FemB proteins, respectively, peptidyl transferases which synthesise the pentaglycine bridges used to crosslink glycan chains in S. aureus ${ }^{9,10}$. During the inner membrane steps of PG synthesis (see Pinho et al. ${ }^{11}$ for a review), the Fem proteins sequentially transfer five glycine residues to the stem peptide of the PG precursor lipid II using glycyl-charged tRNA molecules ${ }^{12}$. Importantly, in vivo and in vitro studies have shown that each Fem protein has strict substrate specificity: FemX adds the first glycine, FemA adds the second and the third and FemB adds the fourth and fifth glycines, and each Fem cannot substitute for another ${ }^{13,14}$. Although $f m h B$ was shown to be an essential gene ${ }^{15}$, mutants carrying transposon inactivated femA or femB grew poorly but were viable, suggesting that $S$. aureus can survive with a PG composed of monoglycine crossbridges ${ }^{9,16}$. However, HPLC analysis of the PG composition in these mutants revealed an overall reduction, but not absence of crosslinked muropeptides.

A second study on the essentiality of $f e m A B$ was done by Strandén and colleagues, who constructed a fem $A B$ mutant, AS145, by allelic replacement of the $f e m A B$ operon by a tetracycline resistance marker ${ }^{17}$. AS145 showed impaired growth, methicillin hypersusceptibility, accumulation of monoglycyl substituted PG monomers and drastically reduced crosslinking of glycan strands, when compared to the parental strain ${ }^{17}$. Cis-complementation of the $f e m A B$ mutation in AS145 with wild-type fem $A B$ restored synthesis of the pentaglycine crossbridge and methicillin resistance, but the growth rate remained low ${ }^{18}$. Therefore the authors postulated that survival of AS145 required compensatory or suppressor mutations ${ }^{18}$. Transcriptional analysis revealed that AS145 underwent severe metabolic adaptations to survive, including upregulation of membrane transporters associated with glycerol uptake (an osmoprotectant), upregulation of the arginine-deiminase pathway (an alternative for ATP production) and alterations in nitrogen metabolism. Collectively these data suggested that $f e m A B$ mutants adapted to survive with shortened crossbridges by reducing metabolic activity to alleviate internal turgor ${ }^{18}$.

The $f e m A B$ operon and the pentaglycine crossbridges are unique features of staphylococci. This makes FemAB proteins potentially interesting targets for MRSA-specific drug design. In this work we wanted to investigate if depletion of the FemAB proteins is lethal in an MRSA strain and to determine the phenotypic defects associated with lack of $f e m A B$ expression.

\section{Results and Discussion}

The femAB operon is essential for the viability of $S$. aureus. Previous $S$. aureus femAB null mutants likely had compensatory mutations ${ }^{16,18,19}$. To evaluate the essentiality of $f e m A B$, as well as the phenotypes resulting from Fem $A B$ depletion in a background without the existence of compensatory mutations, we constructed a conditional fem $A B$ mutant. The $f e m A B$ operon of the clinically relevant CA-MRSA strain MW2 was placed under the control of the IPTG inducible Pspac promoter. As the Pspac promoter is known to be leaky ${ }^{20}$, a plasmid-encoded lacI repressor gene was provided to decrease the basal transcription of $f e m A B$. The resulting strain was named MW2-iFemAB.

Growth of MW2-iFemAB in liquid medium supplemented with IPTG at 0, 10, 25 and $500 \mu \mathrm{M}$ was followed for 10 hours. In the presence of $500 \mu \mathrm{M}$ of IPTG, growth of the conditional mutant was similar to the parental strain MW2 (Fig. 1a), therefore this concentration of inducer was used in subsequent assays. The growth rate of MW2-iFemAB decreased with decreasing IPTG concentrations and, surprisingly, no bacterial growth was observed in the absence of IPTG, indicating that this operon is essential for survival (Fig. 1a), contrarily to what was previously thought. In order to confirm the essentiality of $f e m A B$, we measured colony forming units (CFUs) in cultures of MW2-iFemAB incubated in the absence of IPTG, and found severe loss of viability when compared to IPTG-induced cultures or to parental MW2 (Supplementary Fig. 1). To evaluate the possibility of polar effects on genes downstream of $f e m A B$, we introduced a plasmid encoding $f e m A B$ under the control of the cadmium-inducible promotor Pcad into MW2i-FemAB. Expression of plasmid-encoded fem $A B$ was able to restore growth in cells of MW2-iFemAB background depleted of endogenous femAB (Supplementary Fig. 2). We also tested the essentiality of the femAB operon in an HA-MRSA background - strain COL - and likewise no growth of COL-iFemAB was observed in the absence of IPTG (Supplementary Fig. 3).

To assess the effect of loss of FemAB activity on PG composition, we analysed the cell wall of MW2-iFemAB cells incubated with 500,10 or $0 \mu \mathrm{M}$ of IPTG until bacterial growth was arrested in the non-induced culture (see Methods). As expected, the muropeptide profiles in cells depleted of FemAB show a massive accumulation of peak 4 (Fig. 1b, [IPTG] $0 \mu \mathrm{M}$, see Supplementary Fig. 4 for peak assignment), which was previously identified as the monomeric pentapeptide substituted with a single glycine residue ${ }^{21}$, the substrate of FemA. This was in contrast to cells where femAB expression was fully induced (Fig. 1b, [IPTG] $500 \mu \mathrm{M}$ ), where the major monomeric form present was the pentaglycine substituted monomer (peak 5). To confirm the composition of these muropeptide species, peaks 4 and 5 were analysed by mass spectrometry and found to have molecular weights of 1024.48 Da and 1252.55 Da, respectively, consistent with the expected weight of a monoglycyl- (1024.49 Da) and a pentaglycyl- $(1252.58 \mathrm{Da})$ substituted GlcNAc-MurNAc-pentapeptide. Lack of FemAB also prevented the formation of pentaglycine crosslinked forms such as dimers (peak 11), trimers (peak 15), tetramers (peak 16) and higher order forms which co-elute near the end of the run (Fig. 1b, arrow). Low FemAB expression levels, just enough to sustain bacterial growth ([IPTG] $10 \mu \mathrm{M}$ ), resulted in the presence of some pentaglycine crosslinked forms (peaks 11, 15, 16, etc.). This degree of peptidoglycan structural organisation might be the minimum to ensure cell viability.

Loss of FemAB activity leads to membrane damage. The morphology of cells depleted of FemAB was analysed by super resolution structured illumination microscopy (SIM). MW2-iFemAB was grown with or without IPTG $(500 \mu \mathrm{M})$. Following growth arrest of the non-induced culture (Supplementary Fig. 5, arrow), cells were stained with membrane dye FM 4-64, PG dye Van-FL and DNA dye Hoechst 33342. In the presence of IPTG, MW2-iFemAB cells divided normally with DNA segregation preceding the synthesis of a division septum at mid-cell (Fig. 2a, top row). Cells containing multiple septa were rarely observed $(0.3 \pm 0.6 \%, \mathrm{~N}=300$ septating 
a

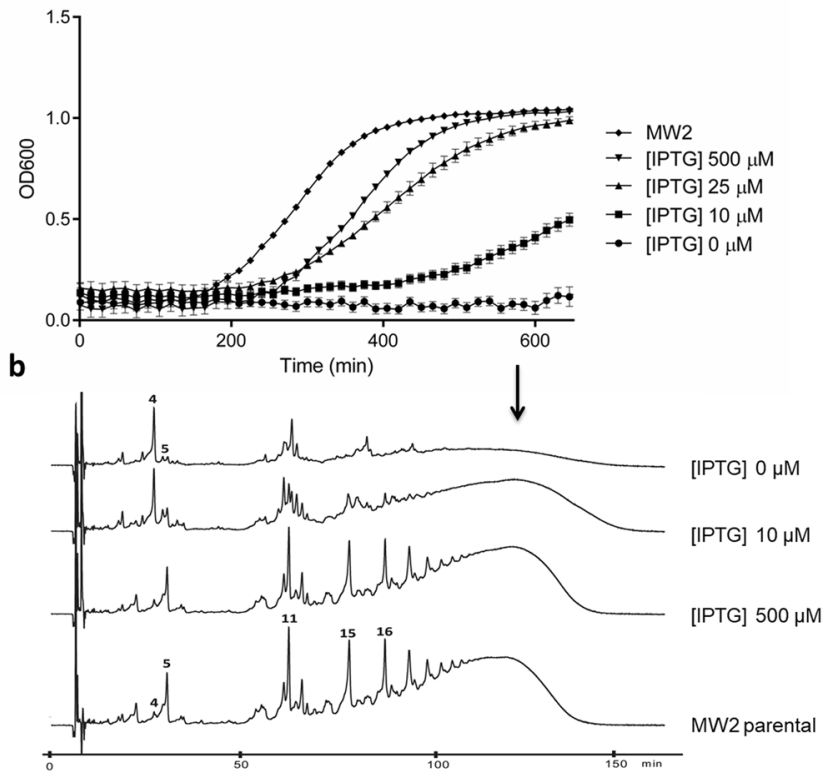

Figure 1. Fem $A B$ are essential for cell viability in S. aureus. (a) Growth curves of parental strain MW2 and strains MW2-iFemAB with IPTG-inducible fem $A B$ operon. In the presence of high IPTG concentrations ([IPTG] $500 \mu \mathrm{M}$ ), growth of MW2-iFemAB was similar to that of MW2. Cell growth was reduced with decreasing IPTG concentrations. In the absence of IPTG ([IPTG] $0 \mu \mathrm{M})$, no cell growth was detected. Symbols indicate means and error bars indicate standard deviation from three biological replicates. (b) Muropeptide HPLC profiles of MW2 and MW2-iFemAB grown in the presence of different levels of IPTG. Depletion of FemAB led to the accumulation of monomeric pentapeptides substituted with one glycine (peak 4), in contrast to pentaglycine forms (peak 5) seen in fully induced ([IPTG] $500 \mu \mathrm{M}$ ) or parental strain (MW2 parental) profiles (see Supplementary Fig. 4 for peak assignment). Loss of FemAB activity also impaired the formation of pentaglycine crosslinked forms such as di-, tri- and tetramers (peaks 11, 15 and 16, respectively) and higher order oligomers (black arrow). Muropeptide profiles shown are representative of three independent experiments.

cells - Fig. 2b, left panel). In contrast, FemAB depleted cells often appeared as pseudomulticellular forms with two or more perpendicular septa $(46.7 \pm 9.9 \%, \mathrm{~N}=300$ septating cells), suggesting that a second round of division starts before daughter cell separation is completed (Fig. 2b, right panel arrows). Furthermore, the nucleoid morphology of Fem AB depleted cells was altered, with the presence of cells containing condensed DNA (Fig. 2a, bottom row asterisks, $43.7 \pm 5.5 \%$ in $\mathrm{FemAB}^{-}$vs $0.7 \pm 0.6 \%$ in $\mathrm{FemAB}^{+}, \mathrm{N}=300$ cells for each condition). Our results are in agreement with previous reports showing that suppressed $\mathrm{fem}$ mutants show irregular placement of cross walls and retarded cell separation ${ }^{16}$. This phenotype can be a consequence of either multiple septation or defective cell splitting.

When cells depleted of FemAB were incubated for longer periods, we noticed a decrease in culture density, suggesting cell lysis (Supplementary Fig. 5). We therefore imaged cells 2 hours after growth arrest and observed extensive membrane damage, characterised by bulges and invaginations (Fig. 3a, arrow, $68.9 \pm 2.0 \%$ in FemAB ${ }^{-}$ vs $4.0 \pm 2.6 \%$ in $\mathrm{FemAB}^{+}, \mathrm{N}=300$ ) and the presence of anucleate cells, indicative of loss of viability (Fig. 3a, asterisks, $48.0 \pm 8.8 \%$ in $\mathrm{FemAB}^{-}$vs $1.0 \pm 1.0 \%$ in $\mathrm{FemAB}^{+}, \mathrm{N}=300$ ). These results suggest that the inability of $S$. aureus to survive with shortened crossbridges could be because the three-dimensional structure of this alternate PG does not confer sufficient robustness and/or flexibility to bear the internal osmotic pressure, in these conditions, causing the cells to rupture. In order to test this hypothesis we incubated MW2-iFemAB in the absence of IPTG (to deplete Fem AB expression) with increasing concentrations of $\mathrm{NaCl}$ added to the medium, to alleviate turgor. MW2-iFemAB was able to grow in the presence of $\mathrm{NaCl}$ in a dose dependent manner (Fig. 3b), confirming that in the absence of pentaglycine crosslinks, the PG layer is not able to withstand the internal pressure exerted on the membrane. Supplementing the medium with a different osmolyte, sucrose, also rescued cells depleted of FemAB (Supplementary Fig. 6). These results are in accordance with data from Hübscher and colleagues ${ }^{18}$, who showed by transcriptome analysis that the fem AB null mutant AS145 adapted to the FemAB deficit by tuning its metabolic pathways, presumably to reduce turgor. It is likely that monoglycyl-substituted muropeptides are not suitable substrates for transpeptidation by $S$. aureus PBPs in vivo and thus crosslinking of glycan chains is stalled after FemAB depletion. Accordingly, solid-state NMR data obtained by Kim et al. ${ }^{22}$ indicated that monoglycyl crossbridges would be too short to connect the glycan chains of the $S$. aureus PG, and that crosslinking with such a reduced bridge length would require a major rearrangement of the tertiary structure of $\mathrm{PG}^{22}$.

FemA activity is not required for its membrane localisation. The Fem proteins are non-ribosomal peptidyl transferases which use dedicated amino acid charged tRNA molecules as substrates, an interesting 
a
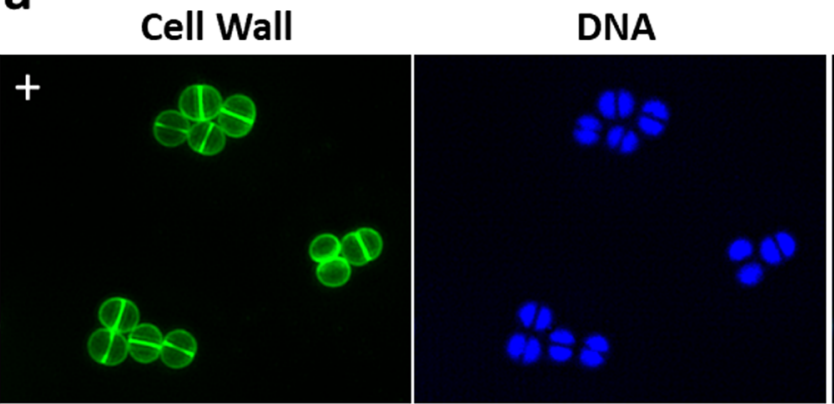

DNA
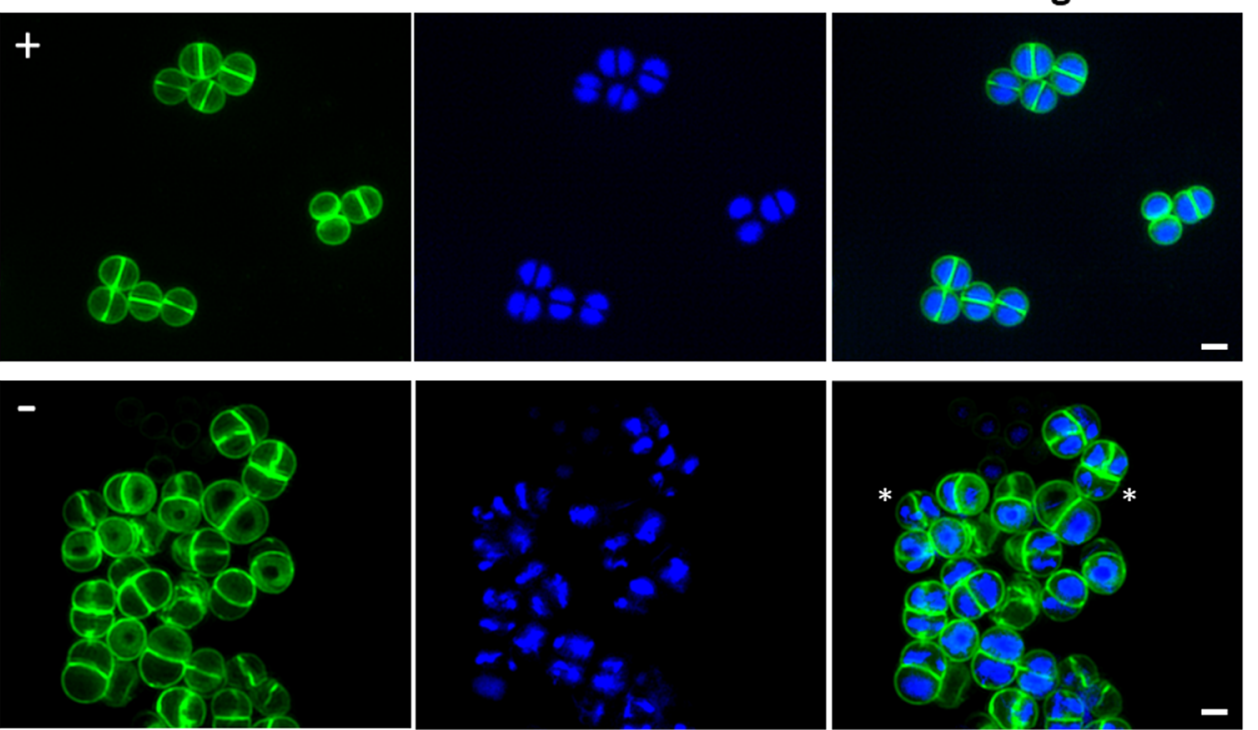

b
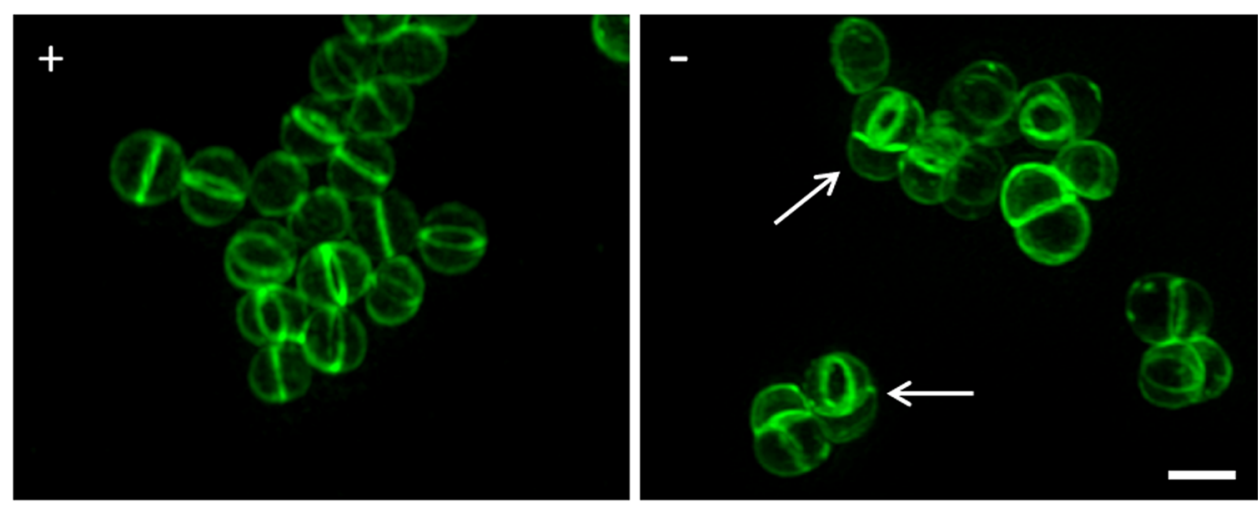

Figure 2. Loss of Fem $A B$ activity inhibits daughter cell separation during division. (a) SIM images of MW2iFemAB cells grown in the presence $(+)$ or absence $(-)$ of IPTG and labelled with cell wall dye Van-FL and DNA dye Hoechst 33342. (b) 3D-SIM projections of MW2-iFemAB cells grown in the presence (+) or absence $(-)$ of IPTG and stained with cell wall dye Van-FL. IPTG-induced cells divide normally with DNA segregation preceding the synthesis of the division septum at mid-cell (Panel (a), top row and Panel (b), left column). In contrast, FemAB depleted cells often had condensed nucleoids (Panel (a), bottom row, asterisks) and appeared as pseudo multicellular forms with two perpendicular septa (Panel (b), white arrows). Scale bars, $1 \mu \mathrm{m}$.

activity seldom seen in nature ${ }^{12}$. The mechanism of this transfer is still poorly understood, as binding sites for entering tRNA molecules have not been identified. In the case of Fem proteins which transfer two amino acids, such as FemA (and FemB), the transfer of both glycines to lipid II appears to occur simultaneously rather than sequentially, judging from in vitro data, which may indicate that these proteins act as homodimers ${ }^{13,23}$. We have recently reported that all three Fem proteins of $S$. aureus localise to the membrane throughout the entire cell cycle, which was unexpected given that these proteins lack canonical transmembrane domains ${ }^{24}$. Therefore a possible mechanism of Fem localisation to the membrane could be through protein activity, which is dependent on interactions with both the substrate lipid II and glycyl-charged tRNA molecules.

In order to investigate the mechanism of localisation of FemA, we identified possible key regions in FemA required for activity, based on the known crystal structure of $\mathrm{FemA}^{25}$ and on homology to the FemX protein from Weisella viridescens ${ }^{26,27}$. We decided to focus on the putative transferase pocket that contains Arg220, Phe224 and Tyr327, which are conserved across the Fem family ${ }^{25,27}$. We also mined the sequence of FemA for regions which could bind DNA/RNA using DP-Bind ${ }^{28,29}$, in order to identify the putative tRNA-binding site. We found that the region with the highest probability of binding to RNA corresponded to the $\alpha 6$ helix (aa 176-188) of FemA, rich in Lys/Arg residues with polar and charged sidechains exposed to the solvent ${ }^{25}$, which could stabilise the entering tRNA. Specifically, amino acids Lys 180 and Arg181 showed $>96 \%$ probability of binding DNA/RNA in each of three individual prediction algorithms performed by DP-Bind (see Methods), and therefore were selected for mutagenesis. 
a
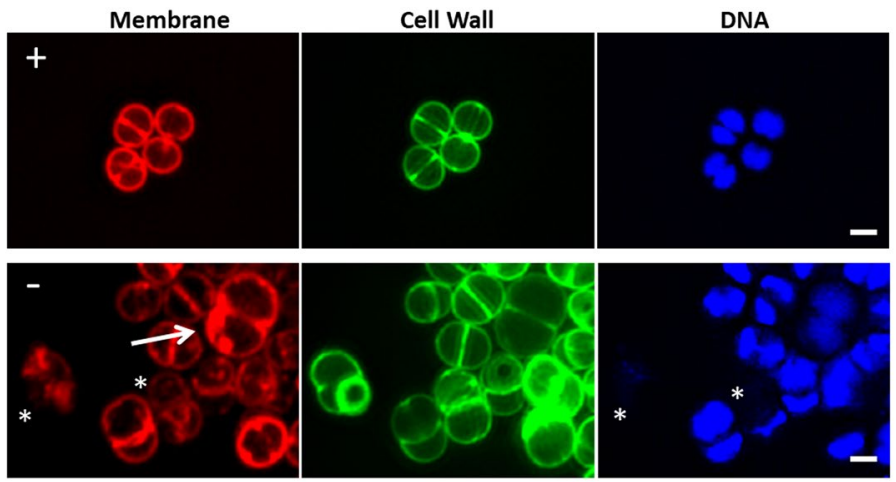

b

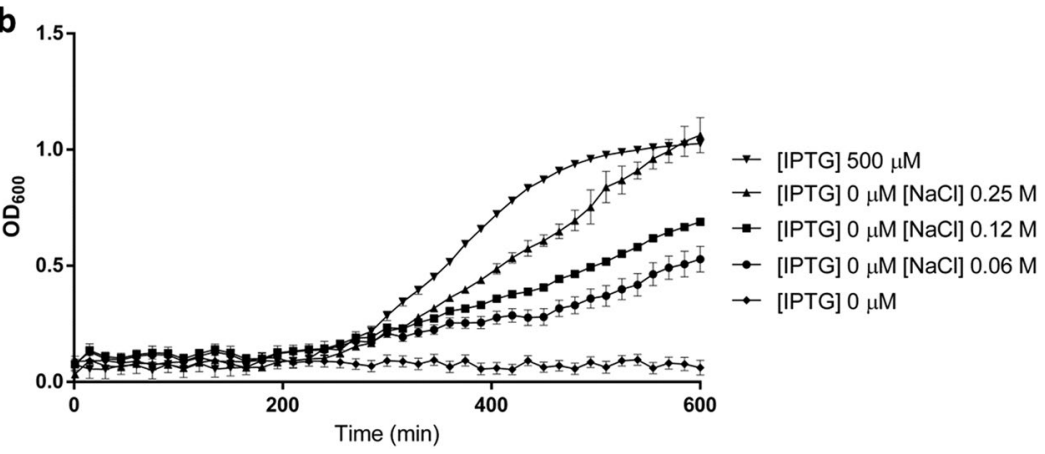

Figure 3. Fem $A B$ activity is required to withstand internal turgor. (a) MW2-iFemAB cells depleted of Fem $A B$ for a period of 2 hours following growth arrest were stained with membrane dye FM 4-64, cell wall dye Van-FL and DNA dye Hoechst 33342 and imaged by SIM. FemAB depleted cells (-) show loss of membrane integrity characterised by bulging and invagination (white arrow) as well as absence of DNA staining (asterisks), indicative of loss of viability, when compared to IPTG-induced cells (+). Scale bars, $1 \mu \mathrm{m}$. (b) Growth rates of MW2-iFemAB incubated in the presence ([IPTG] $500 \mu \mathrm{M}$ ) or absence ([IPTG] $0 \mu \mathrm{M})$ of IPTG, or in the absence of IPTG with increasing $\mathrm{NaCl}$ concentrations. Addition of $\mathrm{NaCl}$ to the medium allowed cells to grow in the absence of FemAB expression, in a dose dependent manner. Symbols indicate means and error bars indicate standard deviation from three biological replicates.

To assess if the selected mutations had an effect on FemA transferase activity, we cloned wild-type femA into the pET-24b expression vector and performed site-directed mutagenesis on femA residues to obtain FemA mutants where the target residues were replaced by alanines. In this way, we constructed $p E T-F e m A^{\mathrm{RF} 220 \mathrm{AA}}$ and $\mathrm{pET} \mathrm{Fem} \mathrm{A}^{\mathrm{Y} 327 \mathrm{~A}}$, in order to express mutants in the transferase domain and $\mathrm{pET}$-FemA ${ }^{\mathrm{KR} 180 \mathrm{AA}}$ to express a mutant of the predicted tRNA binding helix. We purified recombinant FemA ${ }^{\mathrm{wt}}, \mathrm{FemA}^{\mathrm{KR} 180 \mathrm{AA}}, \mathrm{FemA}^{\mathrm{RF} 220 \mathrm{AA}}$ and FemA ${ }^{\mathrm{Y} 327 \mathrm{~A}}$ with C-terminal histidine tags and synthesised the FemA substrate lipid II-Gly ${ }_{1}$ in vitro (see Methods). As recombinant FemA ${ }^{\mathrm{RF} 220 \mathrm{AA}}$ was very unstable and readily precipitated, we could not use it for further assays. Lipid II-Gly 1 was trapped in Triton X-100 micelles and incubated with either FemA ${ }^{\text {wt }}, F_{e m A}{ }^{\mathrm{KR} 180 \mathrm{AA}}$ or FemA ${ }^{\mathrm{Y} 327 \mathrm{~A}}$ in the presence of $\left[\mathrm{U}_{-}{ }^{14} \mathrm{C}\right]$-glycine charged tRNA. After 30, 60 or 90 minutes the lipid fraction was extracted and separated by thin layer chromatography and radioactive glycine transfer to lipid II-Gly ${ }_{1}$ was measured. Both mutants $\mathrm{FemA}^{\mathrm{KR} 180 \mathrm{AA}}$ and $\mathrm{FemA} \mathrm{A}^{\mathrm{Y} 327 \mathrm{~A}}$ transferred less $\left[\mathrm{U}-{ }^{14} \mathrm{C}\right]$-glycine to their substrate than $\mathrm{FemA} \mathrm{A}^{\mathrm{wt}}$, consistent with a reduction of enzyme activity (Fig. $4 \mathrm{a}$ and Supplementary Fig. 7).

In order to both confirm decrease of FemA activity in vivo and study protein localisation, we used the backbone of $\mathrm{pFem}_{\mathrm{AB}}{ }^{\mathrm{wt}}$, a replicative vector encoding a femA-mCherry fusion followed by femB (both under the control of a cadmium inducible promoter), to generate femA-mCherry alleles with the mutations described above. These expression plasmids were transformed into MW2-iFemAB, allowing us to deplete native fem $A B$ expression (in the absence of IPTG) and express mutant alleles (in the presence of cadmium).

We were able to complement the lack of $f e m A B$ expression from the native locus by expressing femA-mCherry-femB from pFemAB ${ }^{\mathrm{wt}}$ in the presence of cadmium $(0.1 \mu \mathrm{M})$, as assessed by growth rates, morphology, lysostaphin (an enzyme that cuts pentaglycine bridges ${ }^{30}$ ) and oxacillin MICs and muropeptide composition (Table 1). Expression of the catalytic site mutants FemA $A^{\mathrm{RF} 20 \mathrm{AA}}$ and $\mathrm{FemA}^{\mathrm{Y} 327 \mathrm{~A}}$ caused a reduction of the pentaglycine substituted monomer content in peptidoglycan (Fig. 4b), although morphology was similar to wild-type and no major differences in lysostaphin and oxacillin MICs were observed (Table 1). In contrast, the double mutation in the $\alpha 6$ helix of FemA caused severe loss of FemA activity. The FemA ${ }^{\mathrm{KR} 180 \mathrm{AA}}$ mutant showed a marked reduction in growth rate, increased lysostaphin and decreased oxacillin resistances and a pseudomulticellular morphology when observed by microscopy (Table 1), similar to what was observed when depleting fem $A B$ expression. Furthermore, analysis of the muropeptide content in this mutant revealed a pronounced accumulation of monoglycyl substituted pentapeptides and concomitant reduction in pentaglycine crosslinked species (Fig. $4 \mathrm{~b}$ and Table 1). Nevertheless, FemA ${ }^{\text {KR180AA }}$ still localised to the membrane, similarly to FemA ${ }^{\text {wt }}$, indicating that FemA localisation is independent of protein activity (Fig. 4c). Because loss of activity in FemA ${ }^{\mathrm{KR} 180 \mathrm{AA}}$ is likely due to a 
a

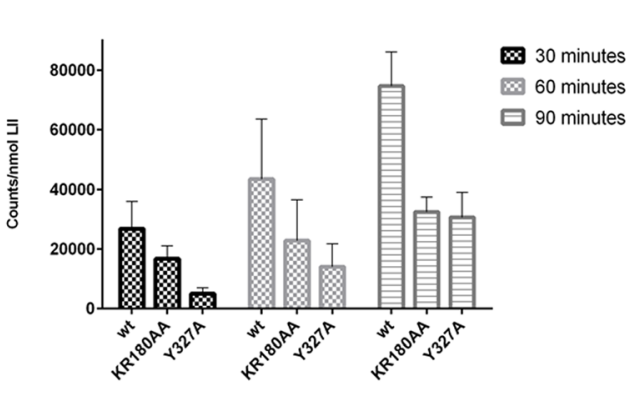

b

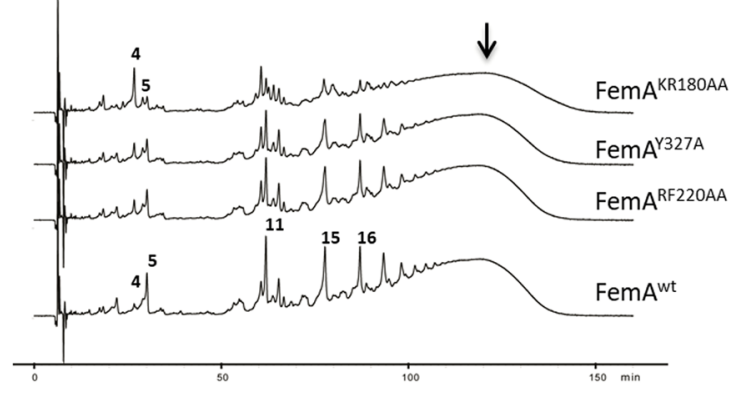

C
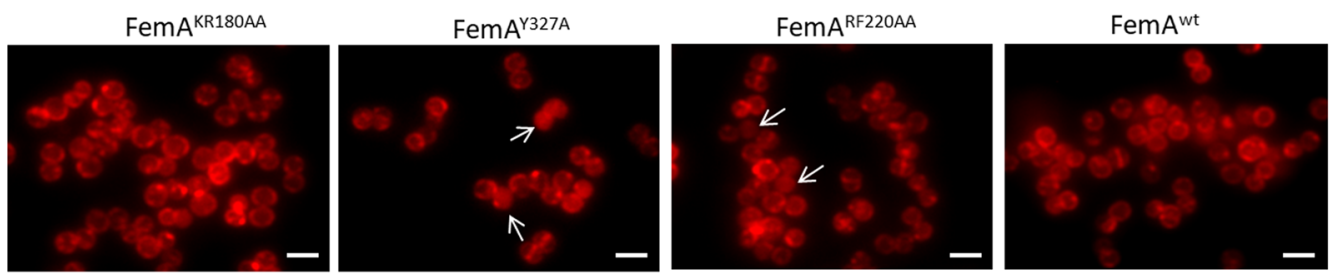

Figure 4. Selected mutations decrease FemA activity. (a) Recombinant FemA ${ }^{\text {wt }}, F e m A^{\mathrm{KR} 180 \mathrm{AA}}$ and FemA ${ }^{\mathrm{Y} 327 \mathrm{~A}}$ were incubated with lipid II-Gly ${ }_{1}$ in the presence of [U- $\left.{ }^{14} \mathrm{C}\right]$-glycine charged tRNA, for either 30, 60 or 90 minutes. Both FemA ${ }^{\mathrm{KR} 180 \mathrm{AA}}$ and $\mathrm{FemA} \mathrm{A}^{\mathrm{Y} 327 \mathrm{~A}}$ showed decreased $\left[\mathrm{U}-{ }^{14} \mathrm{C}\right]$-glycine transfer to lipid II-Gly ${ }_{1}$ when compared to Fem $A^{\text {wt }}$, indicating reduced FemA activity. Columns denote mean values and error bars represent standard deviation from 3 independent experiments. For 90 min samples, $P$ value $=0.013$ for FemA ${ }^{\text {wt }}$ vs Fem $A^{\text {KR180AA }}$ and 0.007 for FemA ${ }^{\text {wt }}$ vs FemA ${ }^{\mathrm{Y} 327 \mathrm{~A}}(\mathbf{b})$ Muropeptide profiles of MW2-iFemAB cells depleted of native Fem $A B$ expression and containing ectopically expressed wild-type FemA-mCherry and FemB $\left(F e m A^{\text {wt }}\right)$ or derivatives with mutations in FemA-mCherry $\left(\mathrm{FemA}^{\mathrm{KR} 180 \mathrm{AA}}, \mathrm{FemA}^{\mathrm{RF} 220 \mathrm{AA}}\right.$ and $\mathrm{FemA}{ }^{\mathrm{Y} 327 \mathrm{~A}}$ ) and wild-type FemB, from the cadmium-inducible promoter Pcad. Ectopic expression of FemA ${ }^{\text {wt }}$ complemented the lack of native FemAB expression, while expression of FemA ${ }^{\mathrm{KR} 180 \mathrm{AA}}$ led to accumulation of monoglycine monomer species (peak 4) with concomitant reduction in higher-order pentaglycine crosslinked species (peaks 11, 15, 16 and black arrow, see Supplementary Fig. 4 for peak assignment). Expression of FemA ${ }^{\mathrm{Y} 327 \mathrm{~A}}$ or FemA ${ }^{\mathrm{RF} 220 \mathrm{AA}}$ led to similar phenotypes, albeit to a lesser extent. (c) Fluorescence microscopy images of strains described in (b). FemA ${ }^{\text {KR180AA }}$ localised to the membrane in $>95 \%$ of the cells, similarly to FemA $A^{\text {wt }}$. FemA $A^{\mathrm{Y} 327 \mathrm{~A}}$ and FemA $\mathrm{AF}^{\mathrm{RF} 20 \mathrm{AA}}$ appeared dispersed in the cytoplasm in a fraction of the population ( $27 \%$ and $10 \%$, respectively, white arrows). $\mathrm{N}=400$ cells for each strain.

\begin{tabular}{|c|c|c|c|c|c|}
\hline & Doubling time (min) & MIC lysostaphin $(\mu \mathrm{g} / \mathrm{ml})$ & MIC oxacillin $(\mu \mathrm{g} / \mathrm{ml})$ & Morphology & Gly5/Gly1 monomer species ratio \\
\hline MW2pFemA ${ }^{\text {KR180AA }}$ & 52 & 2.5 & 0.4 & defective & $1: 3$ \\
\hline MW2pFemA RF220AA $^{\text {Re }}$ & 30 & 0.15 & 1.6 & wt & $2: 1$ \\
\hline MW2pFemA ${ }^{\mathrm{Y} 327 \mathrm{~A}}$ & 27 & 0.08 & 0.8 & wt & $2: 1$ \\
\hline MW2pFemA ${ }^{\mathrm{wt}}$ & 27 & 0.08 & 3.2 & wt & $6: 1$ \\
\hline Parental MW2 & 25 & 0.08 & 1.6 & wt & $6: 1$ \\
\hline
\end{tabular}

Table 1. In vivo activity profiles of FemA mutants.

deficit in tRNA binding, it is possible that FemA ${ }^{\text {KR180AA }}$ could still localise to the membrane through recognition of the lipid-linked peptidoglycan precursor.

The mutations in the substrate binding pocket had a minor effect on FemA localisation, since Fem $\mathrm{A}^{\mathrm{Y} 327 \mathrm{~A}}$ and FemA $\mathrm{A}^{\mathrm{RF} 20 \mathrm{AA}}$ appeared dispersed in the cytoplasm in a fraction of the cell population (Fig. $4 \mathrm{c}$, white arrows). Nevertheless, as these mutations did not seem to decrease protein activity in vivo to a great extent, we could not conclude that the mechanism of FemA localisation to the membrane is via substrate recognition. An alternative possibility is that the recruitment of FemA to the membrane is mediated by protein-protein interactions with the membrane associated eukaryotic-type serine/threonine kinase Stk, a global cell wall synthesis regulator, which was recently shown to interact with FemA and FemB by bacterial two hybrid ${ }^{31}$. However, the same study could not find interactions between Stk and FemX, which initiates Lipid II crossbridge synthesis, or between FemX and FemA $/ \mathrm{B}^{31}$. Further experiments are necessary to clarify the interactions of Fem proteins with each other and with their substrates, in order to understand how the localisation and timing of PG crossbridge synthesis is modulated during the cell cycle. 


\section{Concluding Remarks}

The structural features of the staphylococcal PG seem remarkably unique in nature, as pentaglycine crosslinks have not been observed outside of the genus. These long bridges likely confer high flexibility to S. aureus PG that allows a high level of PG crosslinking, which in turn allows the cell to withstand high internal turgor. Accordingly, $\mathrm{fem} A B$ mutants isolated in the past adapted to life with shortened crossbridges by reducing metabolic activity ${ }^{18}$. Moreover, the nature and length of PG branching has been implicated in resistance to $\beta$-lactams, not only in $S$. aureus, but also in other bacteria such as Streptococcus pneumoniae $e^{7,32-34}$.

We have shown that depletion of FemAB is lethal in CA-MRSA strain MW2 and in HA-MRSA strain COL, leading to the disruption of the cell envelope and causing cells to lose viability. This suggests that monoglycyl-substituted muropeptides are not good substrates for transpeptidation in vivo, either because transpeptidases fail to recognise them or because different $S$. aureus glycan strands are too far apart to be crosslinked via crossbridges with only one glycine.

\section{Methods}

Bacterial growth conditions. Strains and plasmids constructed for this study are listed in Supplementary Table 1. S. aureus strains were grown in tryptic soy broth (TSB, Difco) at 200 r.p.m with aeration at $37^{\circ} \mathrm{C}$ or on tryptic soy agar (TSA, Difco) at 30 or $37^{\circ} \mathrm{C}$. Escherichia coli strains were grown in Luria-Bertani broth (Difco) with aeration, or Luria-Bertani agar (Difco) at 37 or $30^{\circ} \mathrm{C}$. When necessary, antibiotics ampicillin $(100 \mu \mathrm{g} / \mathrm{ml})$, erythromycin $(10 \mu \mathrm{g} / \mathrm{ml})$, kanamycin $(50 \mu \mathrm{g} / \mathrm{ml})$, neomycin $(50 \mu \mathrm{g} / \mathrm{ml})$ or chloramphenicol $(30 \mu \mathrm{g} / \mathrm{ml})$ were added to the media. Unless stated otherwise, isopropyl $\beta$-D-1-thiogalactopyranoside (IPTG, Apollo Scientific) was used at $500 \mu \mathrm{M}$ to induce expression of constructs under the control of the Pspac promoter. Cadmium chloride (SigmaAldrich) was used at $0.1 \mu \mathrm{M}$ when required to induce expression of constructs under the control of the Pcad promoter.

Construction of S. aureus strains. In order to construct an $S$. aureus strain with the femAB operon under the control of an inducible promoter, a fragment containing the first $400 \mathrm{bp}$ of femA was amplified from $S$. aureus MW2 DNA with primer pair spacfemab_P1 EcoRI/spacfemab_P2 BamHI (see Supplementary Table 2 for primer sequences), cut with EcoRI and BamHI restriction enzymes and cloned into pMUTIN4 ${ }^{35}$, downstream of the Pspac promoter, giving plasmid pFem $\mathrm{ABi}$, which was sequenced. pFemABi was then propagated in $\mathrm{DC} 10 \mathrm{~B}$ cells, electroporated into electrocompetent RN4220 cells, and transduced (using phage $80 \alpha$ ) to MW2 and COL, where it integrated in the $f e m A B$ locus by homologous recombination. The resulting strains contain a truncated copy of $f e m A$ under the control of the $f e m A B$ native promoter, and the femAB operon under the control of Pspac. Multicopy plasmid $\mathrm{PMGPII}^{36}$, which encodes Pspac repressor LacI, was then transduced into these strains, giving rise to MW2-iFemAB and COL-iFemAB.

To construct $S$. aureus strains expressing mutated alleles of FemA-mCherry together with wild-type FemB, first a fragment containing femA-mCherry-STOP codon-femB was amplified from pMADfemAmch ${ }^{24}$ using primers pcnfemab_P1 BamHI and pcnfemab_P2 EcoRI. This fragment was cut with BamHI and EcoRI and cloned into replicative vector $\mathrm{pCNX}^{37}$, under the control of $\mathrm{Pcad}$, giving plasmid $\mathrm{pFemAB}^{\mathrm{wt}}$. $\mathrm{pFemAB}^{\mathrm{wt}}$ was then used as the template for site-directed mutagenesis using Phusion polymerase (Thermo Scientific) following manufacturer's instructions. Primers fema_kr180aa_fw/ fema_kr180aa_rev were used to generate pFemA ${ }^{\text {KR180AA }}$, encoding both K180A and R181A mutations; primers fema_rf220aa_fw/fema_rf220aa_rev were used to generate pFemA ${ }^{\mathrm{RF} 220 \mathrm{AA}} \mathrm{B}$, encoding both R220A and F224A mutations; and primers fema_Y327a_fw/fema_Y327a_rev were used to generate $\mathrm{pFem} \mathrm{A}^{\mathrm{Y} 327 \mathrm{~A}} \mathrm{~B}$, encoding the $\mathrm{Y} 327 \mathrm{~A}$ mutation. Each plasmid was sequenced to confirm the presence of the mutations. $p$ Fem $A B^{w t}, p F e m A^{\text {KR180AA }} B, p F e m A^{R F 220 A A} B, p F e m A^{\mathrm{Y} 327 A} B$ and empty vector pCNX were propagated in $\mathrm{DC10B}$, electroporated into RN4220 and transduced to MW2-iFemAB, giving strains MW2pFemAB ${ }^{\text {wt }}$, MW2pFemA ${ }^{\mathrm{KR} 180 \mathrm{AA}}, \mathrm{MW}^{2} \mathrm{pFem}^{\mathrm{RF} 220 \mathrm{AA}}, \mathrm{MW} 2 \mathrm{pFem}^{\mathrm{Y} 327 \mathrm{~A}}$ and MW2pCNX respectively.

Growth curves of S. aureus strains. To assess whether the $f e m A B$ operon is essential for growth, or the effects of FemA mutations on growth rates, overnight cultures of MW2, MW2-iFemAB, COL, COL-iFemAB, MW2pFemAB ${ }^{\text {wt }}, M_{2} 2 p F e m A^{\text {KR180AA }}$, MW2pFemA ${ }^{\text {RF220AA }}$, MW2pFemA ${ }^{\mathrm{Y} 327 \mathrm{~A}}$ were grown in TSB with $500 \mu \mathrm{M}$ of IPTG, with the appropriate antibiotics (when applicable, at the concentrations described above), back-diluted 1:500 in the same medium and grown until the cultures reached an $\mathrm{OD}_{600}$ of 0.7 . At this point the cultures were washed three times to remove IPTG and back-diluted to an $\mathrm{OD}_{600}$ of 0.007 in fresh TSB containing either 0,10 , 25 or $500 \mu \mathrm{M}$ of IPTG, or $0.06,0.12$ or $0.25 \mathrm{M}$ of NaCl, or $0.25 \%$ (w/v) of sucrose, in the case of MW2-iFemAB and COL-iFemAB. In the case of MW2pFemAB ${ }^{\mathrm{wt}}, \mathrm{MW}^{\mathrm{p}} \mathrm{pFem} \mathrm{A}^{\mathrm{KR} 180 \mathrm{AA}}, \mathrm{MW} 2 \mathrm{pFemA}^{\mathrm{RF} 220 \mathrm{AA}}, \mathrm{MW} 2 \mathrm{pFem}^{\mathrm{Y} 327 \mathrm{~A}}$ and MW2pCNX, cells were incubated without IPTG and cadmium chloride was added at $0.1 \mu \mathrm{M}$ to drive the expression of either wild-type or mutant femA alleles from the pCNX-based plasmids.

Growth of all strains was monitored for 10 hours in a Bioscreen C Analyzer (Growth Curves USA), at $37^{\circ} \mathrm{C}$ with shaking with $\mathrm{OD}_{600}$ readings taken every 15 minutes. Growth curves were obtained from three independent experiments done with three biological replicates.

Viability Assays. To assess the effect of $f e m A B$ depletion on $S$. aureus cell viability, overnight cultures of MW2 and MW2-iFemAB grown with IPTG and the appropriate antibiotics were washed three times to remove the IPTG and back-diluted 1:2000 in fresh TSB supplemented with 500 $\mu \mathrm{M}$ of IPTG or no IPTG. Bacterial growth was monitored for 8 hours by measuring $\mathrm{OD}_{600 \mathrm{~nm}}$ and $100 \mu \mathrm{l}$ of appropriate dilutions were plated on TSA (MW2) or TSA supplemented with IPTG at $500 \mu \mathrm{M}$ (MW2-iFemAB), at 1 hour intervals for 8 hours. Following overnight incubation, colony forming units (CFUs) on each plate were counted. In each experiment, CFUs were counted in a total of 6 plates, corresponding to three technical replicates for each of 2 relevant dilutions. These assays were done in triplicate. 
Minimum inhibitory concentration (MIC) assays. MICs of lysostaphin and oxacillin were determined by broth microdilution in sterile 96-well plates. The medium used was TSB, containing a series of two-fold dilutions of each compound. Cultures of $S$. aureus strains and mutants were added at a final density of $\sim 5 \times 10^{5} \mathrm{CFU}$ $\mathrm{ml}^{-1}$ to each well. Wells were reserved in each plate for sterility control (no cells added) and cell viability (no compound added). Plates were incubated at $37^{\circ} \mathrm{C}$. Endpoints were assessed visually after $48 \mathrm{~h}$ and the MIC was determined as the lowest concentration that inhibited growth. All assays were done in triplicate.

Purification and analysis of $S$. aureus muropeptides. To evaluate changes in the peptidoglycan composition caused by the depletion of the $f e m A B$ operon, or caused by the expression of mutant FemA proteins, cells of MW2, MW2-iFemAB, MW2pFemA ${ }^{\text {wt }}, M^{2} 2 p F e m A^{\text {KR180AA }}$, MW2pFemA ${ }^{\text {RF220AA }}$ and MW2pFemA ${ }^{\mathrm{Y} 327 \mathrm{~A}}$ were first grown overnight in TSB supplemented with $500 \mu \mathrm{M}$ of IPTG and the applicable antibiotics. Cultures were then washed three times to remove the IPTG and back-diluted 1:500 in fresh TSB with 0,10 or $500 \mu \mathrm{M}$ of IPTG, in the case of MW2 and MW2-iFemAB, or in the presence of cadmium chloride and absence of IPTG, in the

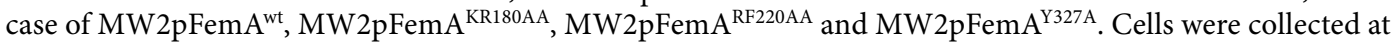
mid-exponential phase and PG was purified as described by Filipe et al. ${ }^{38}$. Muropeptides were prepared from PG samples by digestion with mutanolysin $(0.135 \mathrm{U} / \mu \mathrm{g}$ of PG, from Sigma-Aldrich) and analysed by reverse phase HPLC using a Hypersil ODS (C18) column (Thermo-Fisher Scientific). Muropeptide species were eluted in $0.1 \mathrm{M}$ sodium phosphate, $\mathrm{pH} 2.0$, with a gradient of 5-30\% methanol for 155 minutes and detected at $206 \mathrm{~nm}$. The proportion of pentaglycine to monoglycine substituted monomers (Gly5/Gly1 ratio) in muropeptide preparations was calculated using the areas corresponding to half of the width of peaks 5 (Gly5) and 4 (Gly1) from the obtained chromatogram. To determine the molecular weights of muropeptides in peaks 4 and 5, muropeptide samples were separated by reverse phase HPLC using two Chromolith ${ }^{\circledR}$ Performance RP-8 endcapped 100-4.6 monolithic columns (Merck Millipore) in tandem with a linear gradient from $0.01 \%(\mathrm{v} / \mathrm{v}$ ) Trifluoroacetic acid (TFA) to $0.01 \%$ $(\mathrm{v} / \mathrm{v})$ TFA $15 \%(\mathrm{v} / \mathrm{v})$ acetonitrile over 58 minutes followed by a wash step at $0.01 \%(\mathrm{v} / \mathrm{v})$ TFA $50 \%(\mathrm{v} / \mathrm{v})$ acetonitrile for 6 minutes at a flow of $1 \mathrm{~mL} / \mathrm{min}$ at $30^{\circ} \mathrm{C}$. Sample elution was monitored by recording the absorbance at $206 \mathrm{~nm}$. Muropeptide peaks 4 and 5 were collected, the eluent was evaporated at $30^{\circ} \mathrm{C}$ using a SpeedVac and then resuspended in water, prior to MS analysis by MicroLC-MS using an Eksigent LC4500 coupled to TripleTOF 6600 with Dual spray ion source.

S. aureus imaging by fluorescence microscopy. To evaluate changes in morphology caused by the depletion of the FemAB, or to investigate the localisation of mutant FemA proteins, cells of MW2, MW2-iFemAB, MW2pFemA $A^{\text {wt }}$, MW2pFemA ${ }^{\mathrm{KR} 180 \mathrm{AA}}, \mathrm{MW} 2 \mathrm{pFem}^{\mathrm{RF} 220 \mathrm{AA}}$ and MW2pFemA ${ }^{\mathrm{Y} 327 \mathrm{~A}}$ were first grown overnight in TSB supplemented with $500 \mu \mathrm{M}$ of IPTG and the applicable antibiotics. Cultures were then washed three times to remove the IPTG and back-diluted 1:500 in fresh TSB with 0,25 or $500 \mu \mathrm{M}$ of IPTG, in the case of MW2 and MW2-iFemAB, or in the presence of cadmium chloride and absence of IPTG, in the case of MW2pFemA ${ }^{\text {wt }}$, MW2pFemA ${ }^{\mathrm{KR} 180 \mathrm{AA}}, \mathrm{MW} 2 \mathrm{pFem} \mathrm{A}^{\mathrm{RF} 220 \mathrm{AA}}$ and MW2pFemA ${ }^{\mathrm{Y} 327 \mathrm{~A}}$. Cells were grown to an OD600 nm of 0.4-0.6, collected and then washed with phosphate buffer saline (PBS). In the case of MW2pFemA ${ }^{\text {wt }}, M W 2 p F e m A^{\text {KR180AA, }}$ MW2pFem $A^{\text {RF220AA }}$ and MW2pFemA ${ }^{\mathrm{Y} 327 \mathrm{~A}}$, cells were then mounted on microscope slides covered with a thin layer of $1 \%$ agarose in PBS and imaged by fluorescence microscopy. In the case of MW2 and MW2-iFemAB, to evaluate cell morphology, cells were resuspended in PBS and incubated with membrane dye Nile Red $(10 \mu \mathrm{g} /$ $\mathrm{ml}$, Invitrogen), Hoechst $33342(10 \mu \mathrm{g} / \mathrm{ml}$, Invitrogen) and a mixture containing equal amounts of vancomycin (Sigma) and a BODIPY FL conjugate of vancomycin (Van-FL, Molecular Probes) to a final concentration of $0.8 \mu \mathrm{g} / \mathrm{ml}$, for 5 minutes at room temperature. Cells were then washed three times with PBS before being spotted on the agarose pads and imaged.

Super-resolution Structured Illumination Microscopy (SIM) imaging was performed using an Elyra PS.1 microscope (Zeiss) with a Plan-Apochromat $63 \times / 1.4$ oil DIC M27 objective. SIM images were acquired using five grid rotations, with $34 \mu \mathrm{m}$ grating period for the $561 \mathrm{~nm}$ laser $(100 \mathrm{~mW}), 28 \mu \mathrm{m}$ period for $488 \mathrm{~nm}$ laser $(100 \mathrm{~mW})$ and $23 \mu \mathrm{m}$ period for $405 \mathrm{~nm}$ laser $(50 \mathrm{~mW})$. Images were captured using a Pco.edge 5.5 camera and reconstructed using ZEN software (black edition, 2012, version 8.1.0.484) based on a structured illumination algorithm, using synthetic, channel specific optical transfer functions and noise filter settings ranging from -6 to -8 .

Wide-field fluorescence microscopy was performed using a Zeiss Axio Observer microscope with a Plan-Apochromat $100 \times / 1.4$ oil Ph3 objective. Images were acquired with a Retiga R1 CCD camera (QImaging) using Metamorph 7.5 software (Molecular Devices).

Overexpression and purification of recombinant His-tagged proteins. Recombinant proteins were purified essentially as described by Rohrer et al. ${ }^{39}$, with some modifications. Single colonies of BL21 (DE3) expres-

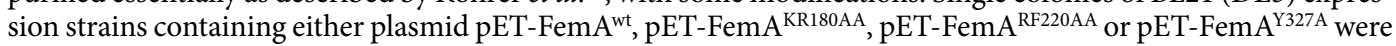
isolated from LA plates with kanamycin and used to inoculate LB $(1 \mathrm{~L})$ containing kanamycin. Cultures were grown to an $\mathrm{OD}_{600 \mathrm{~nm}}$ of approximately 0.6 at which point IPTG was added (final concentration $1 \mathrm{mM}$ ) and incubated for 3 hours with shaking $(150 \mathrm{rpm})$ at $30^{\circ} \mathrm{C}$. Cells were harvested by centrifugation and washed with $50 \mathrm{mM}$ sodium phosphate buffer ( $\mathrm{pH} 7.5$ ) containing $300 \mathrm{mM} \mathrm{NaCl}$ and $20 \%$ glycerol. Afterwards, cells were suspended in the same buffer, containing phenylmethylsulfonyl fluoride (PMSF, final concentration, $0.1 \mathrm{mM}$ ) and lysozyme (final concentration, $1 \mathrm{mg} / \mathrm{mL}$ ), and incubated on ice for $30 \mathrm{~min}$. Cells were then disrupted three times in an ultrasonicator and centrifuged for $30 \mathrm{~min}$ at $4^{\circ} \mathrm{C}$ to precipitate cell debris. The resulting supernatant was purified by affinity chromatography using a Ni-NTA column (Qiagen), following manufacturer's instructions. Protein concentration was assessed using a BCA Protein Assay Kit (Pierce).

Synthesis and purification of lipid II and lipid II-Gly . Lipid II was prepared by reacting undecaprenyl phosphate (Larodan), UDP-MurNAc-pentapeptide from Staphylococcus simulans, UDP-GlcNAc (Sigma) 
and membrane proteins of Micrococcus luteus as previously described ${ }^{13}$. Monoglycyl lipid II was synthesised by reacting lipid I with tRNA preparations, in the presence of enzymes FemX and GlyS, according to the method described by Schneider et al. ${ }^{13}$. Lipid intermediates were extracted from reaction mixtures with an equal volume of butanol/pyridine acetate $(2: 1 ; \mathrm{vol}: \mathrm{vol} ; \mathrm{pH} 4.2)$. Extracts were then purified by anion-exchange chromatography using a Hi-Trap DEAE FF-agarose column (Amersham Biosciences) by reverse-phase HPLC and eluted in a linear gradient from chloroform-methanol-water $(2: 3: 1)$ to chloroform-methanol-300 mM ammonium bicarbonate $(2: 3: 1)$. The fractions containing lipid species were identified by thin layer chromatography with chloroform-methanol-water-ammonia $(88: 48: 10: 1)$ as solvent ${ }^{40}$. The concentration of purified lipids was calculated by measuring inorganic phosphates released after the treatment with perchloric acid, as described previously ${ }^{41}$.

FemA enzymatic activity assay. In order to compare the activity of wild-type FemA to selected FemA mutants, enzymatic reactions were performed as described previously ${ }^{13}$. Briefly, $100 \mu \mathrm{l}$ reactions were prepared containing $2.5 \mathrm{nmol}$ of lipid II-Gly $1,10 \mu \mathrm{g}$ of glycyl-tRNA synthetase (GlyS), $25 \mu \mathrm{g}$ of tRNA, $2 \mathrm{mM}$ ATP and 50 $\mathrm{nmol}\left[{ }^{14} \mathrm{C}\right]$-glycine in Tris buffer $(100 \mathrm{mM}$ Tris- $\mathrm{HCl}, 20 \mathrm{mM} \mathrm{MgCl} 2, \mathrm{pH} 7.5$, and $0.8 \%$ Triton X-100). Then $2.7 \mu \mathrm{g}$ of wild-type FemA or FemA mutant protein was added and the reaction mixtures were incubated for 30, 60 or 90 minutes at $30^{\circ} \mathrm{C}$. Lipid intermediates were then extracted and analysed by thin layer chromatography, as described above. Finally, the amount of $\left[{ }^{14} \mathrm{C}\right]$-glycine transferred to lipid II-Gly was quantified using phosphoimaging in a STORM system (GE Healthcare). Enzymatic assays were done in triplicate.

Identification of FemA residues possibly involved in tRNA binding. Identification of FemA residues which could bind glycyl-charged tRNA was performed using DP-Bind ${ }^{28,29}$ (http://lcg.rit.albany.edu/dp-bind/), a sequence-based web server which predicts DNA/RNA binding domains in proteins based on biochemical properties of amino acids and evolutionary information. Probability maps were generated using PSI-BLAST position-specific scoring matrix (PSSM) and three distinct machine learning methods that use evolutionary information: support vector machine (PSSM-SVM), kernel logistic regression (PSSM-KLR), and penalized logistic regression (PSSM-PLR). FemA residues K180 and R181 were identified as possibly part of DNA-binding domains based on strict consensus between the three methods. SwissPdb viewer/Deep view (http://www.expasy. org/spdbv/) was used to evaluate the structure of FemA, using file 1LRZ (doi: 10.2210/pdb1LRZ/pdb) deposited in the RCSB PDB by Benson et al.

\section{Data Availability}

Source data are available from the corresponding author upon reasonable request.

\section{References}

1. Chambers, H. F. \& DeLeo, F. R. Waves of resistance: Staphylococcus aureus in the antibiotic era. Nat Rev Micro 7, 629-641 (2009).

2. Grundmann, H., Aires-de-Sousa, M., Boyce, J. \& Tiemersma, E. Emergence and resurgence of meticillin-resistant Staphylococcus aureus as a public-health threat. LANCET 368, 874-885 (2006).

3. Schleifer, K. \& Kandler, O. Peptidoglycan types of bacterial cell walls and their taxonomic implications. Bacteriol Rev 36, 407-477 (1972).

4. Vollmer, W., Blanot, D. \& de Pedro, M. Peptidoglycan structure and architecture. FEMS Microbiol Rev 32, 149-167 (2008).

5. Hartman, B. \& Tomasz, A. Low-affinity penicillin-binding protein associated with beta-lactam resistance in Staphylococcus aureus. J Bacteriol 158, 513-516 (1984).

6. Berger-Bachi, B. Insertional inactivation of staphylococcal methicillin resistance by Tn551. J Bacteriol 154, 479-487 (1983).

7. de Lencastre, H. et al. Antibiotic resistance as a stress response: complete sequencing of a large number of chromosomal loci in Staphylococcus aureus strain COL that impact on the expression of resistance to methicillin. Microb Drug Resist 5, 163-175 (1999).

8. de Lencastre, H. \& Tomasz, A. Reassessment of the number of auxiliary genes essential for expression of high-level methicillin resistance in Staphylococcus aureus. Antimicrob Agents Chemother 38, 2590-2598 (1994).

9. Berger-Bächi, B., Barberis-Maino, L., Strässle, A. \& Kayser, F. FemA, a host-mediated factor essential for methicillin resistance in Staphylococcus aureus: molecular cloning and characterization. Mol Gen Genet 219, 263-269 (1989).

10. Rohrer, S., Ehlert, K., Tschierske, M., Labischinski, H. \& Berger-Bächi, B. The essential Staphylococcus aureus gene fmhB is involved in the first step of peptidoglycan pentaglycine interpeptide formation. Proc Natl Acad Sci USA 96, 9351-9356 (1999).

11. Pinho, M. G., Kjos, M. \& Veening, J. W. How to get (a)round: mechanisms controlling growth and division of coccoid bacteria. Nat Rev Microbiol 11, 601-614 (2013).

12. Berger-Bächi, B. \& Tschierske, M. Role of fem factors in methicillin resistance. Drug Resist Updat 1, 325-335 (1998).

13. Schneider, T. et al. In vitro assembly of a complete, pentaglycine interpeptide bridge containing cell wall precursor (lipid II-Gly5) of Staphylococcus aureus. Mol Microbiol 53, 675-685 (2004).

14. Ehlert, K., Schröder, W. \& Labischinski, H. Specificities of FemA and FemB for different glycine residues: FemB cannot substitute for FemA in staphylococcal peptidoglycan pentaglycine side chain formation. J Bacteriol 179, 7573-7576 (1997).

15. Tschierske, M. et al. Identification of three additional femAB-like open reading frames in Staphylococcus aureus. FEMS Microbiol Lett 171, 97-102 (1999)

16. Henze, U., Sidow, T., Wecke, J., Labischinski, H. \& Berger-Bächi, B. Influence of femB on methicillin resistance and peptidoglycan metabolism in Staphylococcus aureus. J Bacteriol 175, 1612-1620 (1993).

17. Strandén, A., Ehlert, K., Labischinski, H. \& Berger-Bächi, B. Cell wall monoglycine cross-bridges and methicillin hypersusceptibility in a fem $A B$ null mutant of methicillin-resistant Staphylococcus aureus. J Bacteriol 179, 9-16 (1997).

18. Hubscher, J. et al. Living with an imperfect cell wall: compensation of femAB inactivation in Staphylococcus aureus. BMC Genomics 8, 307 (2007).

19. de Jonge, B. et al. Altered muropeptide composition in Staphylococcus aureus strains with an inactivated femA locus. J Bacteriol 175, 2779-2782 (1993).

20. Yansura, D. G. \& Henner, D. J. Use of the Escherichia coli lac repressor and operator to control gene expression in Bacillus subtilis. Proc Natl Acad Sci USA 81, 439-443 (1984).

21. de Jonge, B. L., Chang, Y. S., Gage, D. \& Tomasz, A. Peptidoglycan composition of a highly methicillin-resistant Staphylococcus aureus strain. The role of penicillin binding protein 2A. J Biol Chem 267, 11248-11254 (1992).

22. Kim, S. J., Chang, J. \& Singh, M. Peptidoglycan architecture of Gram-positive bacteria by solid-state NMR. Biochim Biophys Acta 1848, 350-362 (2015).

23. Rohrer, S. \& Berger-Bachi, B. FemABX peptidyl transferases: a link between branched-chain cell wall peptide formation and betalactam resistance in gram-positive cocci. Antimicrob Agents Chemother 47, 837-846 (2003). 
24. Monteiro, J. M. et al. Peptidoglycan synthesis drives an FtsZ-treadmilling-independent step of cytokinesis. Nature 554, 528-532 (2018).

25. Benson, T. et al. X-ray crystal structure of Staphylococcus aureus FemA. Structure 10, 1107-1115 (2002).

26. Biarrotte-Sorin, S. et al. Crystal structures of Weissella viridescens FemX and its complex with UDP-MurNAc-pentapeptide: insights into FemABX family substrates recognition. Structure 12, 257-267 (2004).

27. Maillard, A. et al. Structure-based site-directed mutagenesis of the UDP-MurNAc-pentapeptide-binding cavity of the FemX alanyl transferase from Weissella viridescens. J Bacteriol 187, 3833-3838 (2005).

28. Hwang, S., Gou, Z. \& Kuznetsov, I. B. DP-Bind: a web server for sequence-based prediction of DNA-binding residues in DNAbinding proteins. Bioinformatics 23, 634-636 (2007).

29. Kuznetsov, I. B., Gou, Z., Li, R. \& Hwang, S. Using evolutionary and structural information to predict DNA-binding sites on DNAbinding proteins. Proteins 64, 19-27 (2006).

30. Francius, G., Domenech, O., Mingeot-Leclercq, M. \& Dufrêne, Y. Direct observation of Staphylococcus aureus cell wall digestion by lysostaphin. J Bacteriol 190, 7904-7909 (2008).

31. Jarick, M. et al. The serine/threonine kinase Stk and the phosphatase Stp regulate cell wall synthesis in Staphylococcus aureus. Sci Rep 8, 13693 (2018).

32. Filipe, S. \& Tomasz, A. Inhibition of the expression of penicillin resistance in Streptococcus pneumoniae by inactivation of cell wall muropeptide branching genes. Proc Natl Acad Sci USA 97, 4891-4896 (2000).

33. Filipe, S., Severina, E. \& Tomasz, A. The role of murMN operon in penicillin resistance and antibiotic tolerance of Streptococcus pneumoniae. Microb Drug Resist 7, 303-316 (2001).

34. Fiser, A., Filipe, S. \& Tomasz, A. Cell wall branches, penicillin resistance and the secrets of the MurM protein. Trends Microbiol 11, $547-553(2003)$

35. Vagner, V., Dervyn, E. \& Ehrlich, S. D. A vector for systematic gene inactivation in Bacillus subtilis. Microbiology 144, 3097-3104 (1998).

36. Pinho, M. G., Filipe, S. R., de Lencastre, H. \& Tomasz, A. Complementation of the essential peptidoglycan transpeptidase function of penicillin-binding protein 2 (PBP2) by the drug resistance protein PBP2A in Staphylococcus aureus. J Bacteriol 183, 6525-6531 (2001).

37. Monteiro, J. M. et al. Cell shape dynamics during the staphylococcal cell cycle. Nat Commun 6, 8055 (2015).

38. Filipe, S. R., Tomasz, A. \& Ligoxygakis, P. Requirements of peptidoglycan structure that allow detection by the Drosophila Toll pathway. EMBO Rep 6, 327-333 (2005).

39. Rohrer, S. \& Berger-Bächi, B. Application of a bacterial two-hybrid system for the analysis of protein-protein interactions between FemABX family proteins. Microbiology 149, 2733-2738 (2003).

40. Rick, P. D. et al. Characterization of the lipid-carrier involved in the synthesis of enterobacterial common antigen (ECA) and identification of a novel phosphoglyceride in a mutant of Salmonella typhimurium defective in ECA synthesis. Glycobiology 8 , 557-567 (1998).

41. Rouser, G., Fkeischer, S. \& Yamamoto, A. Two dimensional then layer chromatographic separation of polar lipids and determination of phospholipids by phosphorus analysis of spots. Lipids 5, 494-496 (1970).

\section{Acknowledgements}

This study was funded by the European Research Council through grant ERC-2017-CoG 771709 (to M.G.P.), by Project LISBOA-01-0145-FEDER-007660 Microbiologia Molecular, Estrutural e Celular (to ITQB-NOVA), by the German Research Foundation (DFG; SCHN1284/1-2) to T.S. and FCT fellowship SFRH/BD/71993/2010 (J.M.M.). Mass spectrometry data of muropeptides was acquired by the Mass Spectrometry Unit (UniMS), ITQB/ iBET, Oeiras, Portugal.

\section{Author Contributions}

J.M.M., H.G.S. and M.G.P. designed the research. J.M.M. constructed all strains and performed all experiments with the exception of the glycine incorporation assays, which were performed by D.R. and muropeptide purification for mass spec analysis and viability curves, which were performed by G.C. J.M.M., G.C., D.R., T.S., S.R.F. and M.G.P. analysed the data. J.M.M. and M.G.P. wrote the manuscript.

\section{Additional Information}

Supplementary information accompanies this paper at https://doi.org/10.1038/s41598-019-41461-1.

Competing Interests: The authors declare no competing interests.

Publisher's note: Springer Nature remains neutral with regard to jurisdictional claims in published maps and institutional affiliations.

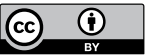

Open Access This article is licensed under a Creative Commons Attribution 4.0 International License, which permits use, sharing, adaptation, distribution and reproduction in any medium or format, as long as you give appropriate credit to the original author(s) and the source, provide a link to the Creative Commons license, and indicate if changes were made. The images or other third party material in this article are included in the article's Creative Commons license, unless indicated otherwise in a credit line to the material. If material is not included in the article's Creative Commons license and your intended use is not permitted by statutory regulation or exceeds the permitted use, you will need to obtain permission directly from the copyright holder. To view a copy of this license, visit http://creativecommons.org/licenses/by/4.0/.

(C) The Author(s) 2019 